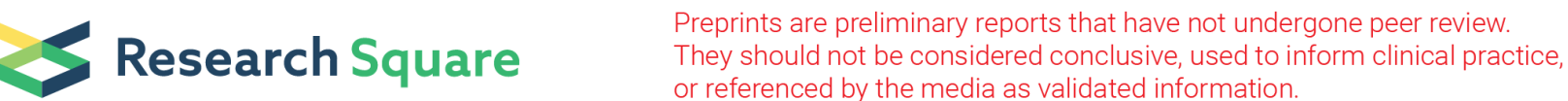

\section{Ensemble of Nucleic Acid Absolute Quantitation Modules for Accurate Copy Number Variation Detection and Targeted RNA Profiling}

\section{Lucia Wu}

Rice University https://orcid.org/0000-0001-5051-9685

Peng Dai

Rice University https://orcid.org/0000-0002-4581-3473

\section{Michael Wang}

Rice University https://orcid.org/0000-0001-7009-6958

\section{Sherry Chen}

Rice University

\section{Evan Cohen}

The University of Texas MD Anderson Cancer Center https://orcid.org/0000-0003-4192-9644

\section{Gitanjali Jayachandran}

U.T. M.D. Anderson Cancer Center

Jinny Zhang

NuProbe USA Inc.

\section{Angela Serrano}

NuProbe USA Inc.

Naoto Ueno

M. D. Anderson Cancer Center https://orcid.org/0000-0002-0166-7275

\section{JAMES REUBEN}

UT MD ANDERSON CANCER CENTER

\section{Carlos Barcenas}

The University of Texas MD Anderson Cancer Center

David Zhang ( $\square$ genomic.dave@gmail.com )

Rice University

\section{Article}

Keywords: droplet digital PCR, copy number variation, quantitative amplicon sequencing

Posted Date: September 28th, 2021

DOI: https://doi.org/10.21203/rs.3.rs-923491/v1 
License: (c) (i) This work is licensed under a Creative Commons Attribution 4.0 International License. Read Full License

Version of Record: A version of this preprint was published at Nature Communications on April 4th, 2022. See the published version at https://doi.org/10.1038/s41467-022-29487-y. 


\section{Abstract}

Current gold standard for absolute quantitation of a specific DNA sequence is droplet digital PCR (ddPCR), which has been applied to gene copy number variation (CNV) detection. However, the number of quantitation modules in ddPCR has been limited by fluorescence channels, which thus limits the CNV sensitivity due to sampling error following Poisson distribution. Here we develop a PCR-based molecular barcoding NGS approach, quantitative amplicon sequencing (QASeq), for accurate absolute quantitation which is compatible with high multiplexing to include over 200 quantitation modules. By attaching barcodes to individual target molecules with high efficiency, 2-plex QASeq exhibits higher and more consistent conversion yield than ddPCR in absolute molecule count quantitation. Multiplexed QASeq improves CNV sensitivity to allow confident distinguishment of 2.05 ploidy from normal 2.00 ploidy. We applied multiplexed QASeq to serial longitudinal plasma cfDNA samples from patients with metastatic ERBB2+ (HER2+) breast cancer seeking association with tumor progression. We further show an RNA QASeq panel for targeted expression profiling on a wide range of RNA samples including tumor formalinfixed, paraffin-embedded (FFPE) RNA.

\section{Introduction}

Quantitation of specific nucleic acid sequences is the basis of many important biological applications; one example is gene ploidy calculation for detection of copy number variations $(\mathrm{CNVs})^{1-3}$, which is one of the most frequently observed genetic biomarker types in cancer ${ }^{4,5}$. It is present in $3 \%-98 \%$ of tumor cases depending on the cancer type ${ }^{6}$, and is clinically relevant as prognostic markers and as therapeutic targets $^{7-9}$. Additionally, quantitation of specific RNA molecules for gene expression patterns profiling reflects the state of a cell or tissue $\mathrm{e}^{10,11}$ and may reveal pathological mechanisms underlying diseases ${ }^{12-}$ 14.

Droplet digital PCR (ddPCR) is the gold standard for absolute quantitation of specific nucleic acid sequences $^{15,16}$. The quantitation precision enabled small fold change measurements in CNV detection. The minimum copy number gain that can be distinguished from normal ploidy of 2.0 was improved from 3.0 using quantitative PCR (qPCR) to approximately 2.4 using $\mathrm{ddPCR}^{17}$, rendering ddPCR useful for CNV detection in clinical settings ${ }^{18}$. However, improved CNV sensitivity is still highly desired especially for cellfree DNA (cfDNA) samples in which tumor DNA (ctDNA) are significantly "diluted" by DNA from normal tissues ${ }^{19,20}$. The physical limitation from stochasticity in molecule sampling leads to actual number of observed DNA molecules and thus the observed ploidy deviating from the expected "true value" in CNV quantitation (Supplementary Fig. S1). Poisson statistics can be used to model this sampling process: the standard deviation of a Poisson variable $X$ is $\sqrt{X}$, and the coefficient of variation (CV) is ${ }^{\frac{1}{\sqrt{X}}}$. Thus, in principle, increasing the DNA input amount or the number of genomic sites to quantify in the same gene would improve the limit of detection (LoD). Because the DNA input is usually limited especially in plasmaderived cfDNA, to overcome the Poisson distribution problem, ensemble of quantitation modules to 
sample a large number of independent genomic loci on the same gene is required to further improve CNV sensitivity. Highly multiplexed ddPCR remains challenging due to limited fluorescence channels.

As an alternative approach to ddPCR, we present Quantitative Amplicon Sequencing (QASeq), a PCRbased molecular barcoding NGS approach for accurate absolute quantitation which is compatible with high multiplexing. Herein, we demonstrate that 2-plex QASeq exhibited higher and more consistent conversion yield than ddPCR in absolute molecule count quantitation, and enables CNV quantitation accuracy similar to ddPCR. Multiplexed QASeq improves LoD to allow confident distinguishing 2.05 ploidy from normal 2.00 ploidy, and is applied to longitudinal serial 57 plasma cfDNA samples from patients with metastatic ERBB2+ (HER2+) breast cancer. Finally, an RNA QASeq panel covering 20 genes are demonstrated on a wide range of RNA samples including tumor and placenta FFPE RNA.

\section{Results}

QASeq development and 2-plex demonstration. Unique molecular identifiers (UMIs) ${ }^{21,22}$ are attached to individual input DNA strand via two cycles of PCR with long annealing time for high and uniform barcoding efficiency. After further amplification, NGS reads originating from the same input DNA strand carry the same UMI sequence and thus belong to the same UMI family. Therefore, the unique UMI family count represents the number of input DNA strands (Fig. 1a).

We demonstrated QASeq for absolute quantitation and copy number calculation using a 2-plex panel containing 2 quantitation modules in gene ERBB2 (target) and EIF2C1 (reference) respectively (Supplementary Sect. 2 and Sect. 6), and compared with ddPCR for the same 2 genes side-by-side using five replicated experiments for both methods (Fig. 1b). QASeq exhibited higher and more consistent conversion yield than ddPCR, where conversion yield is the fraction of input molecules that are observed in the experiment. $10 \mathrm{ng}$ Human PBMC gDNA from the same healthy donor was used per experiment, corresponding to 2,790 haploid copies. QASeq showed higher conversion yield ( $86 \%$ on average) than ddPCR ( $53 \%$ on average). The coefficient of variation (CV) of molecule count was lower for QASeq (5.0\% for ERBB2, 2.5\% for EIF2C1) than for ddPCR (12.8\% for ERBB2, 13.3\% for EIF2C1) in 5 replicates.

High dynamic range of DNA input was observed for absolute quantitation using 2-plex QASeq (Fig. 1c). Observed ERBB2 molecule counts by QASeq were close to expected value calculated from DNA input amount because of high conversion yield. Lower conversion yield at $1 \mathrm{ng}$ input DNA was possibly a result of material loss at low concentration.

ERBB2 ploidy calculated from QASeq was accurate and highly reproducible (Fig. 1d). ERBB2 ploidy was calculated as $2 \times$ ERBB2 molecule counts / EIF2C1 molecule counts. The mean of 5 replicates was 1.98, which is close to normal ploidy of 2.00 . The CV for calculated ERBB2 ploidy was $4.8 \%$ in five replicates. Spike-in cell-line DNA samples with different expected ERBB2 ploidy were assayed by 2-plex QASeq and ddPCR, and high correlation in calculated ploidy was observed between the methods (Supplementary Fig. S2). 
The histogram for observed UMI family size distribution followed log-normal distribution after removal of small families (Fig. 1e), because in theory random yield difference in PCR efficiency was amplified exponentially in PCR cycles post UMI attachment. Large number of UMI families with UMI family size $<3$ were observed. These small UMI families were not used for molecule counting as they are likely results of polymerase and sequencing errors.

Highly multiplexed QASeq for CNV detection. Combination of multiple absolute quantitation modules for CNV detection was demonstrated to overcome the stochasticity in sampling. Experimentally, we did observe that the stochastic error in copy number quantitation reduced as a function of number of quantitation modules in the gene (Fig. 2a). A QASeq panel with 175 modules was designed for ERBB2 CNV detection, with 49 quantitation modules in ERBB2 and 123 modules in other regions of human genome serving as the reference (Supplementary Sect. 3 and Sect. 6). The rest of 3 modules are in Chromosome $\mathrm{X}$ thus are not used in $\mathrm{CNV}$ analysis. Five technical replicates were conducted with $8.3 \mathrm{ng}$ healthy PBMC gDNA per library. 1 to 49 modules were used for ERBB2 ploidy quantitation. The CV of ERBB2 ploidy in 5 replicated experiments was reduced from $3.25-0.69 \%$ as the number of modules increases, consistent with theory based on Poisson distribution.

Combination of multiple quantitation modules in QASeq allowed confident discrimination between 2.05 and 2.00 ploidy ERBB2 samples using QASEq. 2.05 ploidy sample was prepared by mixing a normal PBMC DNA sample and ERBB2-positive cell line (SK-BR-3) DNA. Normal sample was tested in quadruplicates, and 2.05 ploidy sample was tested in duplicates.

Multiplexed QASeq for CNV in tumor tissue samples. QASeq was applied to 18 fresh/frozen (FF) tumor samples from 16 breast cancer patients and was compared with both ddPCR and immunohistochemistry $(\mathrm{IHC})$ results on $E R B B 2$. QASeq $E R B B 2$ ploidy results were concordant with $\mathrm{IHC}$ and ddPCR from the tumor tissue (Fig. 2cd) with potentially fewer false positives. ERBB2 ploidy from QASeq showed high correlation with ddPCR (Supplementary Fig. S3). For the single sample with discordance between QASeq and IHC, ddPCR agreed with QASEq. For all the 3 samples with discordance between QASeq and ddPCR, ddPCR ploidy were between 2.5 and 3 and IHC agreed with QASeq for negative call. There was no case where IHC and ddPCR agreed on a call that conflicted with QASeq results.

175-plex QASeq is theoretically equivalent to $C(175,2)=15225$ different ddPCR CNV assays (Supplementary Fig. S4). To utilize quantitation modules beyond just calculating ERBB2 ploidy, modules in 'reference' were further grouped based on gene. Ploidy for 10 genes and 2 chromosomal regions were calculated from QASEq. To reduce the false positives of CNV calls in clinical samples and account for potential poor sample quality, sequential Mann-Whitney $U$ tests on each gene of interest was performed (Supplementary Fig. S5, see Supplementary Sect. 3 on data analysis for multiplexed QASeq with $>2$ quantitation modules). As an example, ploidy of each of the 175 quantitation modules for a normal PBMC DNA sample and for an FF DNA sample from breast cancer tumor section was shown in Fig. 2e. In clinical sample analysis, the ploidy values for a gene will be reported as 2.00 if there is no statistical difference between gene of interest and reference by Mann-Whitney $U$ test. We summarized the CNV 
results in all of $18 \mathrm{FF}$ samples (Fig. 2f). The LoD for ERBB2 CNV was calculated from the five technical replicates in healthy gDNA to be 1.97 ploidy for copy number loss and 2.04 for gain (Supplementary Table S2 and Supplementary Sect. 3). Additionally, ERBB2 ploidy in PBMC DNA from 10 different healthy donors were assessed with 175-plex QASeq and ddPCR respectively for biological variability (Supplementary Fig. S6). There is no sample with ploidy deviating from 2.00 for over $10 \%$, but ddPCR showed wider ploidy range ( 1.8 to 2.1 ) than QASeq ( 1.9 to 2.0$)$ in the 10 normal samples.

QASeq could improve clinical sensitivity in CNV assessment. The ploidy values of all observed gene ploidies in 18 tumor DNA samples are plotted as a histogram (Supplementary Fig. S7). Using methods with LoD at 1.6 and 2.4 ploidy, such as ddPCR, $44 \%$ of the CNVs may be missed. Additionally, QASeq allows quantitation of mutations down to $0.1 \%$ variant allele frequency (VAF) with UMI error correction (Supplementary Fig. S8). As an NGS-based quantitation method, sequence mutation calling is performed in addition to the copy number calculation. We designed QASeq panel amplicons to include hot spot mutations commonly observed in breast cancer (Supplementary Sect. 4 and Sect. 6).

\section{Study of QASeq liquid biopsy results with disease progression in ERBB2+ (HER2+) metastatic breast} cancer patients. QASeq breast cancer liquid biopsy panel (Supplementary Sect. 6) was applied to serial longitudinal plasma cell-free DNA (cfDNA) samples and was compared with disease progression. 57 plasma cfDNA samples from 15 patients with ERBB2 + metastatic breast cancer were tested by QASeq, with 2-8 samples per patient, where all patients had a baseline sample obtained at the moment of diagnosis of metastatic breast cancer, and follow-up samples were obtained at different timepoints for each patient. All patients had a diagnosis of ERBB2+ (IHC II+ or III + or $\mathrm{FISH}+)$ metastatic breast cancer and the $E R B B 2$ status was confirmed from a biopsy obtained from tumor tissue.

We summarized ERBB2 ploidy change in cfDNA and disease progression dates for each patient using a swimmer plot (Fig. 3a). There were 8 patients who developed disease progression who had a plasma sample collected within 6 months before or after the disease progression. ERBB2 amplification or increase of $E R B B 2$ ploidy relative to the previous time point was observed in 6 out of the 8 patients who developed disease progression. In the other two patients (de-identified patient ID 2697 and 2366), disease progression was reported 4 times for each patient and abnormal ERBB2 CNV can only explain part of the disease progression. Significant allele frequency changes in PIK3CA G1049R mutation in patient 2697 and in SNP rs1309838194 in patient 2366 were correlated with disease progression respectively (Fig. 3b). PIK3CA G1049R mutation (COSV55874453) is considered to be structural damaging alteration as disease-causing drivers ${ }^{23,24}$ in breast cancer. The VAF for PIK3CA G1049R in circulating cfDNA was increased by over 10-fold during follow-up, serving as evidence for increased tumor fraction. The PIK3CA mutation may have contributed to disease progression. In patient 2366, VAF for the SNP rs1309838194 in ERBB2 changed from around $50 \%$ (heterozygous at baseline time point) to $80 \%$ during follow-up, which indicated increased tumor-derived DNA in plasma and may be associated to disease progression. Since the overall ERBB2 ploidy in plasma was still normal, we think that the copy-neutral loss of heterozygosity $(\mathrm{LOH})^{25,26}$ may be present in the tumor, leading to SNP allelic imbalance. Taken the CNV and mutation 
results together, abnormal change was reported by QASeq in all the 8 patients who developed disease progression.

The 15 cases were classified according to whether or not there was abnormal molecular findings in QASeq liquid biopsy and whether or not disease progression was clinically observed (Fig. 3c). Chi-square test suggested that the QASeq result and progression are not statistically independent $(p=0.038)$. Although all cases with disease progression were featured with QASeq abnormal findings showing good sensitivity, increase of ERBB2 ploidy was also observed in 4 patients who did not develop disease progression within this time frame. Because all patients presented here were treated with ERBB2-targeted therapy trastuzumab and pertuzumab until disease progression, molecular change may not translate to clinically observed disease progression in all cases.

Furthermore, tumor ERBB2 ploidy was inferred from plasma QASeq results and was compared with FISH. Because both CNV and mutation information are available from QASeq, tumor fraction in plasma cfDNA can be estimated based on VAF of tumor mutation; tumor ploidy can be calculated with plasma ploidy and tumor fraction. We demonstrated this normalization in two cases (Fig. 3d), where pathogenic mutation was observed in baseline cfDNA with VAF between $1 \%$ and $30 \%$. Tumor FISH results were collected at three time points in case 1834. QASeq detected ERBB2 amplification in plasma cfDNA 5 months earlier than FISH from tumor tissue. In addition, the inferred tumor ERBB2 ploidy by QASeq was consistent with the available tumor ploidy from FISH. The inferred tumor ERBB2 ploidy was generally stable in both of the two patients, so ERBB2 ploidy change in cfDNA was influenced by tumor fraction. Based on the correlation of QASeq results with progression and FISH results, we envision non-invasive and sensitive longitudinal study of CNV and mutation change in plasma by QASeq can help with understanding disease progression and resistance mechanism.

RNA QASeq for gene expression level quantitation. Next, we demonstrated QASeq technology for RNA quantitation in a variety of samples including tumor tissue FFPE RNA, total blood RNA and total liver RNA. RNA sample was reverse transcribed to CDNA as input for QASEq. Random hexamer was chosen as reverse transcription primer to be compatible with low-quality fragmented FFPE RNA. A targeted multigene breast cancer panel covering 78 amplicons in 15 cancer-related and 5 reference genes similar to Oncotype DX ${ }^{27}$ panel was built (Supplementary Sect. 5 and Sect. 6). Expression of each gene is calculated from the molecule count of each amplicon, based on UMI count and conversion yield, and is further normalized relative to the expression level of the 5 reference genes in log2 scale (Supplementary Fig. S9).

The RNA quantitation accuracy was firstly validated using ERCC RNA spike-in mix. 16 ERCC sequences were targeted with 16 amplicons. The ERCC RNA sample was diluted and mixed with commercial human total liver RNA for a final expected molecule count between 3 and 100,000. The observed molecule count showed good correlation with the expected (Fig. 4a). QASeq quantitation for RNA was across five orders of magnitude and as few as 3 expected molecules were detected. 
Reproducibility for expression level relative to reference genes was evaluated. Total liver RNA was assayed with breast cancer panel in replicates and consistent expression level was observed (Fig. 4b). We observed that multiple quantitation modules (amplicons) per gene reduced quantitation variability in expression level. The standard deviation for relative expression level in triplicate experiments became lower as the number of amplicons per gene increased from 1 to 5 (Fig. 4c), with median standard deviation reduced from 0.44 to 0.21 . Outlier was only observed when only 1 amplicon is considered.

RNA Expression level from QASeq was extensively compared with other technologies including RNAseq ${ }^{28}$, NanoString nCounter ${ }^{29}$, Microarray ${ }^{30}$ and RT-qPCR using FFPE RNA from breast cancer and lung cancer tissue. The expression level was normalized in the same way relative to the 5 reference genes for all the methods, and was summarized in Fig. $5 d$ for a breast cancer FFPE RNA. RNA QASeq is consistent with RNAseq and NanoString nCounter. Microarrary, however, showed poor correlation with any of the other methods. RNA QASeq was further compared with these technologies in a couple other samples (Supplementary Fig. S10-13). Nanostring showed high correlation with QASeq in all samples, but required much higher input amount than RNA QASEq. Low expression level species are dropped out at $10 \mathrm{ng}$ as compared to the typical $150 \mathrm{ng}$ input (Supplementary Fig. S14). Microarray showed poor concordance with both QASeq and nanostring in all samples (Supplementary Fig. S13 and S15). QASeq was consistent with RNAseq in most samples. However, because RNAseq was a non-targeted approach, most reads were wasted on genes of no interest and coverage uniformity issue led to poor robustness for the quantitation of lowly expressed genes as it was observed in the two FFPE samples (Supplementary Fig. S11). RT-qPCR is consistent with UMI-based QASeq quantitation, but is limited by low multiplexing ability.

We summarized the relative expression level in four clinical FFPE and 3 normal placenta FFPE samples (Fig. 4d). Hierarchical clustering indicated the expression patterns were the most similar between normal placenta samples.

\section{Discussion}

QASeq developed in this work provides an accurate absolute nucleic acid quantitation method that can be conveniently scaled up to high multiplexing, thus overcoming sampling error from Poisson distribution for CNV detection. We demonstrated confident distinguishment of 2.05 ploidy from 2.00 ploidy. Absolute quantitation is based on a highly efficient PCR-based barcoding approach. Based on Poisson distribution theory and our observation, QASeq allows the construction of targeted panels with adjustable CNV sensitivity for each gene by changing the number of amplicons that cover each gene of interest.

Robust, consistent conversion yield and high number of quantitation modules are significant for QASEq. We chose to perform two cycles of PCR-based barcoding with long annealing time, because the fraction of DNA molecules in a sample represented in the final NGS library is low for ligation-based UMI attachment. As the number of primer pairs increases for a multiplex PCR amplification, there is a combinatorial explosion of potential primer dimers and non-specific genomic amplification. The dimer problem is more complicated when UMls must be incorporated in the multiplex PCR. By combining 
simulated annealing design using dimer likelihood estimation (SADDLE) ${ }^{31}$, a primer set optimization software developed in our lab, with a nested protocol, about $60 \%$ on-target rate is maintained even in the liquid biopsy panel with 223 modules.

The most accessible sample type for cancer monitoring is cfDNA derived from plasma. However, CNV monitoring using cfDNA is understudied comparing to mutation detection ${ }^{32}$. Common CNV detection methods Immunohistochemistry and in situ hybridization are limited to tissue/cell samples, and not applicable to non-invasive cfDNA. Other cfDNA-compatible methods including ddPCR, NGS panels such as FoundationOne and Guardant 360, and microarray are not sensitive enough, all requiring $>25 \%$ heterozygous single copy loss or gain for detection which corresponds to 1.75 ploidy for loss and 2.25 ploidy for gain. The high CNV sensitivity of QASeq may allow better clinical sensitivity of copy number changes.

As a proof-of-concept demonstration, QASeq is used to infer tumor gene ploidy from cfDNA when tumor mutation is observed in two clinical cases. A single gene ploidy number in cfDNA may not be actionable because it is decided by both circulating tumor DNA (ctDNA) fraction and CNV in cancer cells, not to reflect any of the two explicitly. As an example, $1 \%$ tumor fraction in cfDNA with tumor copy number of 20 , or $6 \%$ tumor fraction with tumor copy number of 5 has the same cfDNA overall ploidy (2.18), but the former may have better outcome to HER2-targeted therapy. Deconvolution of tumor CNV from cfDNA will be complicated when tumor tissue is highly heterogeneous. Clonal mutation, the mutation that is present in all cancer cells within tumors, needs to be identified in this case to infer the average gene ploidy in tumor.

We identified normal ERBB2 ploidy but significant SNP allelic imbalance in ERBB2 in one patient, which may be due to copy-neutral LOH in tumor. QASeq breast cancer panel covers hotspot cancer mutations while designing multiple regions in gene of interest so not many SNPs are included. Inspired by this case study, we can intentionally add quantitation modules for SNP calling in gene of interest to better identify allelic imbalance for higher confidence of $\mathrm{LOH}$ detection.

\section{Methods}

Oligonucleotides and Reagents. All oligonucleotides were purchased from Integrated DNA Technologies (100uM in IDTE, pH 8.0). Oligonucleotide sequences are provided in Supplementary Section 6. Primers in the 2-plex QASeq panel are dual-HPLC purified; primers in other panels are standard-desalted. Phusion High-Fidelity DNA polymerase and deoxynucleoside triphosphates (dNTPs) were purchased from New England Biolabs. PowerUp SYBR Green Master Mix was purchased from Thermo Fisher Scientific. iTaq Universal SYBR Green Supermix was puchased from Bio-Rad Laboratories. AMPure XP was purchased from Beckman Coulter. NGS index primers (NEBNext Multiplex Oligos for Illumina) was purchased from New England Biolabs. 
QASeq protocol. Library preparation consists of three PCR reactions: UMI PCR, nested PCR and index PCR, all performed on a T100 Thermal Cycler (Bio-Rad). In UMI PCR, DNA sample was mixed with $1 \mathrm{U}$ Phusion High-Fidelity DNA polymerase, Phusion HF buffer, forward and outer reverse primers (15 nM each), and dNTPs ( $0.2 \mathrm{mM}$ each) to reach a total volume of $50 \mu \mathrm{L}$.

Thermal cycling started with $30 \mathrm{~s}$ at $98{ }^{\circ} \mathrm{C}$, followed by 2 cycles of $10 \mathrm{~s}$ at $98{ }^{\circ} \mathrm{C}, 30 \mathrm{~min}$ at $63{ }^{\circ} \mathrm{C}$ and $15 \mathrm{~s}$ at $72{ }^{\circ} \mathrm{C}$, and then 2 cycles of $10 \mathrm{~s}$ at $98{ }^{\circ} \mathrm{C}, 15 \mathrm{~s}$ at $63^{\circ} \mathrm{C}$ and $15 \mathrm{~s}$ at $72{ }^{\circ} \mathrm{C}$, finally 5 cycles of $10 \mathrm{~s}$ at $98{ }^{\circ} \mathrm{C}$ and $30 \mathrm{~s}$ at $71^{\circ} \mathrm{C}$. During the last $5 \mathrm{~min}$ of the second $30 \mathrm{~min}$ at $63^{\circ} \mathrm{C}, 1.5 \mu \mathrm{M}$ of each universal primer was added while keeping the reactions inside the thermal cycler. After UMI PCR, 1.6X AMPure XP beads purification was performed.

In nested PCR, the eluate from the previous step was mixed with PowerUp SYBR Green Master Mix (1X final concentration) and $15 \mathrm{nM}$ each inner reverse primer. Thermal cycling started with 3 min at $95^{\circ} \mathrm{C}$, followed by 2 cycles of $10 \mathrm{~s}$ at $95^{\circ} \mathrm{C}$ and $30 \mathrm{~min}$ at $60{ }^{\circ} \mathrm{C}$. The PCR product was purified by $1.6 \mathrm{X}$ AMPure $\mathrm{XP}$ beads.

Next, index PCR was performed; the eluate from the previous step was mixed with iTaq Universal SYBR Green Supermix ( $1 \mathrm{X}$ final concentration) and $250 \mathrm{nM}$ each NEBNext index primers. Thermal cycling started with a 3 min incubation step at $95{ }^{\circ} \mathrm{C}$, followed by 25 cycles of $10 \mathrm{~s}$ at $95^{\circ} \mathrm{C}$ and $30 \mathrm{~s}$ at $65{ }^{\circ} \mathrm{C}$, and finally 2 min at $65^{\circ} \mathrm{C}$. After index PCR, double-side size selection ( $0.4 \mathrm{X}, 0.4 \mathrm{X}$ ratio) was performed. Libraries were normalized and loaded onto an Illumina sequencer.

DNA extracted from FF or blood samples was sheared to 150 bp peak length using Covaris LE220 Focused Ultrasonicator before library preparation.

In RNA QASeq, RNA sample was firstly reverse transcribed to cDNA as input for QASeq protocol. RNA was mixed with dNTP $(0.5 \mathrm{mM})$, Murine RNase Inhibitor (8 U), M-MuLv buffer (1X), M-MuLV Reverse Transcriptase $(8 \mathrm{U})$, and random hexamer $(6 \mu \mathrm{M})$. The mixture was incubated at $25^{\circ} \mathrm{C}$ for 5 mins, at $42{ }^{\circ} \mathrm{C}$ for $60 \mathrm{mins}$, and then inactivated at $65^{\circ} \mathrm{C}$ for 20 mins. The reaction mixture was directly used as input for UMI PCR without purification.

Sequencing was performed on HiSeq or NextSeq (Illumina) with 2X 150 bp paired-end reads and dual 8 bp index.

NGS data processing. NGS adapter sequences were first removed from FASTQ data using custom Python code; alignment was performed using Bowtie2 software ${ }^{33}$. UMI grouping and CNV analysis were performed using custom Matlab code; detailed description of the algorithm can be found in Supplementary Section 2 and Section 3. Mutation analysis was performed using custom Python and Matlab code; detailed description can be found in Supplementary Section 4.

Digital droplet PCR. ddPCR CNV Assays from Bio-Rad were used in this study. Specifically, ddPCR Copy Number Assay: ERBB2, Human (Fluorophore: FAM, UniqueAssayID: dHsaCP1000116) and ddPCR Copy 
Number Assay: AG01 (EIF2C1), Human (Fluorophore: HEX, UniqueAssayID: dHsaCP2500349) were purchased. Reaction setup, thermal cycling conditions and data acquisition were performed according to Bio-Rad protocol for ddPCR Copy Number Variation Assays. $10 \mathrm{ng}$ of input DNA were used for each reaction.

Samples. Fresh frozen (FF) breast tissue samples from breast cancer patients were purchased from OriGene Technologies. ERBB2 status of the tumor tissue measured by immunohistochemistry (IHC) was obtained from the vendor. Genomic DNA from FF samples and buffy coat of blood samples was extracted using QIAamp DNA Mini (Qiagen) following manufacture's protocol.

56 plasma samples from 15 ERBB2+ metastatic breast cancer patients in de-identified format were collected from MD Anderson Cancer Center. All procedures performed in studies involving human participants were approved by Institutional Review Board at MD Anderson (protocols PA16-0507 and PA19-0375), and were in accordance with the 1964 Helsinki declaration and its later amendments or comparable ethical standards. Informed consent was obtained from all participants.

Cell-free DNA was extracted from plasma using QIAamp MinElute ccfDNA Mini Kit (Qiagen) following manufacture's protocol. Samples were quantified by qPCR with Human cell-line gDNA NA18537 as reference. The concentration calculated from qPCR reflects the amplifiable DNA.

Normal human placenta FFPE was purchased from BioChain. Total RNA from FFPE was extracted using RNeasy FFPE Kit (Qiagen). Human liver total RNA was purchased from Takara Bio. Human whole blood samples from healthy people were purchased from Zen-Bio Inc. RNA from fresh total blood was extracted using Monarch Total RNA Miniprep Kit (New England BioLabs).

ERBB2-positive cell line (SK-BR-3) DNA was from the National Institute of Standards and Technology (NIST) Standard Reference Material 2373 (ATCC).

Infer tumor ERBB2 ploidy from plasma. cfDNA ERBB2 Ploidy = mean tumor ERBB2 Ploidy * tumor fraction $\%+2.0$ * (1 - tumor fraction\%); tumor fraction $\%$ = mutation VAF in cfDNA / VAF in tumor. We took pathogenic mutation observed in baseline cfDNA with VAF between $1 \%$ and $30 \%$ for tumor fraction calculation, to avoid the influence of SNP. Baseline mutation in 2 patients (1834 and 3669) were identified. We hypothesized mutation VAF in pure tumor is $50 \%$ (monoallelic), so that tumor fraction $\%=$ mutation VAF in cfDNA * 2 .

RNAseq. Library preparation was performed using NEBNext Ultra II RNA Library Prep Kit for Illumina. Ribosomal RNA depletion was performed using NEBNext rRNA Depletion Kit v2. Raw fastq reads were initially quality filtered using Trimmomatic v0.39. Specifically, individual reads were trimmed to the longest continuous segment for which phred quality score (Q) was $\geq 20(Q \geq 20$ represents $\sim 99 \%$ accuracy per nucleotide position). Reads shorter than $50 \mathrm{bp}$ after trimming were discarded. Next, libraries were aligned to the human reference genome (GRCh38) using bowtie2 v2.4.4. After alignment, sam files were sorted and converted to bam files using samtools v1.12. HTseqv0.13.5 in mode 'intersection-strict' 
and with additional parameter '-minaqual 1 ' was used to estimate the number of reads that mapped to each gene of interest. Finally, StringTie v2.1.7 was used to calculate TPM-normalized gene abundance.

Nanostring and Microarray. Extracted RNA samples were sent to Amsbio LLC for Nanostring test using nCounter Breast Cancer 360 V2 Panel, and were sent to UT Southwestern Medical Center Microarray Core Facility for GeneChip Human Transcriptome Array 2.0 (HTA 2.0) test.

\section{Declarations}

Data availability: The main data supporting the results in this study are available within the paper and its Supplementary Information. Source data are provided with this paper. Raw sequencing data can be found at https://doi.org/10.6084/m9.figshare.16529640

Code availability: NGS data analysis pipeline for QBDA variant calling is available from Github (https://github.com/wrj915/QASeq).

Acknowledgements: This work was supported by NIH awards U01CA233364 and R01CA203964 to D.Y.Z., and CPRIT award RP180147 to D.Y.Z.

Author contributions: L.R.W. P.D., and D.Y.Z. conceived the project. L.R.W. and P.D. designed and conducted the experiments, and analyzed the data. M.X.W. performed experiments and analyzed the data. S.X.C. analyzed the data. J.X.Z. and A.V.S. performed RNA QASeq experiments. E.N.C., G.J., N.T.U., J.M.R., and C.H.B. provided clinical plasma samples and analyzed the data. L.R.W., P.D., C.H.B. and D.Y.Z. wrote the paper with input from all authors.

Competing interests: There are patents pending on the QASeq method. P.D., L.R.W., S.X.C., and M.X.W. declares a competing interest in the form of consulting for Nuprobe USA. D.Y.Z. declares a competing interest in the form of consulting for and significant equity ownership in Nuprobe USA, Torus Biosystems, and Pana Bio.

\section{References}

1. Freeman, J. L. et al. Copy number variation: new insights in genome diversity. Genome Res. 16, 949961 (2006). 
2. Conrad, D. F. et al. Origins and functional impact of copy number variation in the human genome. Nature 464, 704-712 (2010).

3. Zarrei, M., MacDonald, J. R., Merico, D. \& Scherer, S. W. A copy number variation map of the human genome. Nat. Rev. Genet. 16, 172-183 (2015).

4. Shlien, A. \& Malkin, D. Copy number variations and cancer. Genome Med. 1, 1-9 (2009).

5. Matsui, A., Ihara, T., Suda, H., Mikami, H. \& Semba, K. Gene amplification: mechanisms and involvement in cancer. Biomol. Concepts 4, 567-582 (2013).

6. Ciriello, G. et al. Emerging landscape of oncogenic signatures across human cancers. Nat. Genet. 45, 1127-1133 (2013).

7. Nahta, R., Yu, D., Hung, M.-C., Hortobagyi, G. N. \& Esteva, F. J. Mechanisms of disease: understanding resistance to HER2-targeted therapy in human breast cancer. Nat. Clin. Pract. Oncol. 3, 269-280 (2006).

8. Nielsen, D. L., Andersson, M. \& Kamby, C. HER2-targeted therapy in breast cancer. Monoclonal antibodies and tyrosine kinase inhibitors. Cancer Treat. Rev. 35, 121-136 (2009).

9. Wang, Q., Yang, S., Wang, K. \& Sun, S.-Y. MET inhibitors for targeted therapy of EGFR TKI-resistant lung cancer. J. Hematol. Oncol. 12, 1-11 (2019).

10. Whitehead, A. \& Crawford, D. L. Variation in tissue-specific gene expression among natural populations. Genome Biol. 6, 1-14 (2005).

11. Lamant, L. et al. Gene-expression profiling of systemic anaplastic large-cell lymphoma reveals differences based on ALK status and two distinct morphologic ALK + subtypes. Blood 109, 21562164 (2007).

12. Bertucci, F. et al. Gene expression profiling of colon cancer by DNA microarrays and correlation with histoclinical parameters. Oncogene 23, 1377-1391 (2004).

13. Cooper-Knock, J. et al. Gene expression profiling in human neurodegenerative disease. Nat. Rev. Neurol. 8, 518-530 (2012).

14. Petty, R. D., Nicolson, M. C., Kerr, K. M., Collie-Duguid, E. \& Murray, G. I. Gene expression profiling in non-small cell lung cancer: from molecular mechanisms to clinical application. Clin. Cancer Res. 10, 3237-3248 (2004).

15. Hindson, B. J. et al. High-throughput droplet digital PCR system for absolute quantitation of DNA copy number. Anal. Chem. 83, 8604-8610 (2011).

16. White III, R. A., Quake, S. R. \& Curr, K. Digital PCR provides absolute quantitation of viral load for an occult RNA virus. J. Virol. Methods 179, 45-50 (2012).

17. Whale, A. S. et al. Comparison of microfluidic digital PCR and conventional quantitative PCR for measuring copy number variation. Nucleic Acids Res. 40, e82-e82 (2012).

18. Heredia, N. J. et al. Droplet Digital ${ }^{T M}$ PCR quantitation of HER2 expression in FFPE breast cancer samples. Methods 59, S20-S23 (2013). 
19. Moss, J. et al. Comprehensive human cell-type methylation atlas reveals origins of circulating cellfree DNA in health and disease. Nat. Commun. 9, (2018).

20. Snyder, M. W., Kircher, M., Hill, A. J., Daza, R. M. \& Shendure, J. Cell-free DNA Comprises an in Vivo Nucleosome Footprint that Informs Its Tissues-Of-Origin. Cell 164, 57-68 (2016).

21. Kinde, I., Wu, J., Papadopoulos, N., Kinzler, K. W. \& Vogelstein, B. Detection and quantification of rare mutations with massively parallel sequencing. Proc. Natl. Acad. Sci. 108, 9530-9535 (2011).

22. Newman, A. M. et al. Integrated digital error suppression for improved detection of circulating tumor DNA. Nat. Biotechnol. 34, 547-555 (2016).

23. Chen, L. et al. Characterization of PIK3CA and PIK3R1 somatic mutations in Chinese breast cancer patients. Nat. Commun. 9, 1-17 (2018).

24. Martínez-Sáez, O. et al. Frequency and spectrum of PIK3CA somatic mutations in breast cancer. Breast Cancer Res. 22, 1-9 (2020).

25. Beroukhim, R. et al. Inferring loss-of-heterozygosity from unpaired tumors using high-density oligonucleotide SNP arrays. PLoS Comput. Biol. 2, e41 (2006).

26. O’Keefe, C., McDevitt, M. A. \& Maciejewski, J. P. Copy neutral loss of heterozygosity: a novel chromosomal lesion in myeloid malignancies. Blood, J. Am. Soc. Hematol. 115, 2731-2739 (2010).

27. Paik, S. et al. A multigene assay to predict recurrence of tamoxifen-treated, nodenegative breast cancer. N. Engl. J. Med. 351, 2817-2826 (2004).

28. Consortium, S. A comprehensive assessment of RNA-seq accuracy, reproducibility and information content by the Sequencing Quality Control Consortium. Nat. Biotechnol. 32, 903 (2014).

29. Kulkarni, M. M. Digital multiplexed gene expression analysis using the NanoString nCounter system. Curr. Protoc. Mol. Biol. 94, 25B-10 (2011).

30. Tan, P. K. et al. Evaluation of gene expression measurements from commercial microarray platforms. Nucleic Acids Res. 31, 5676-5684 (2003).

31. Zhang, D. et al. Designing Highly Multiplex PCR Primer Sets with Simulated Annealing Design using Dimer Likelihood Estimation (SADDLE). Prepr. Res. Sq. https//doi.org/10.21203/rs.3.rs-491811/v1.

32. Frampton, G. M. et al. Development and validation of a clinical cancer genomic profiling test based on massively parallel DNA sequencing. Nat. Biotechnol. 31, 1023-1031 (2013).

33. Langmead, B. \& Salzberg, S. L. Fast gapped-read alignment with Bowtie 2. Nat. Methods 9,357 (2012).

\section{Figures}




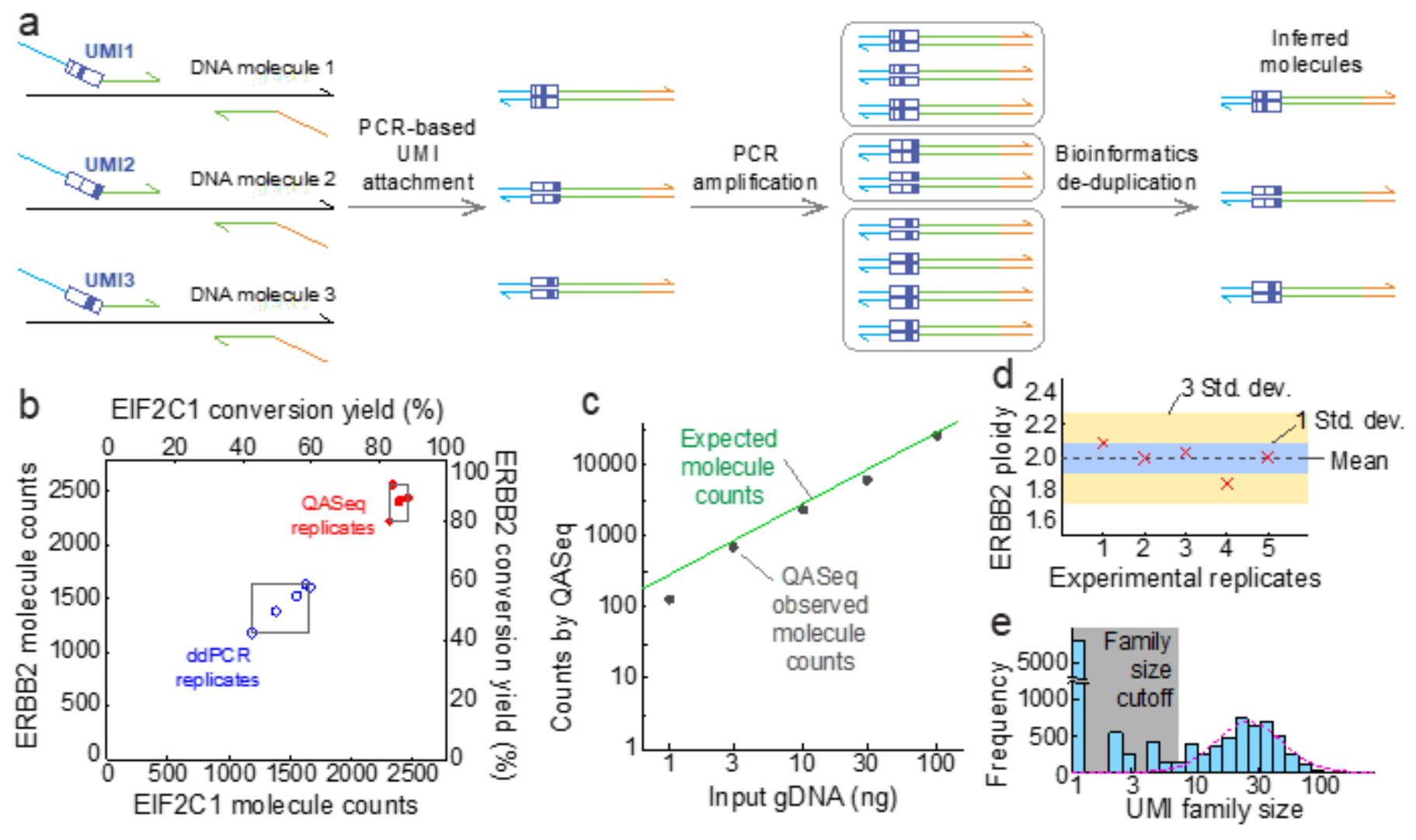

\section{Figure 1}

NGS-based QASeq DNA absolute quantitation modules. a. Schematic of a single QASeq module for quantitation of DNA bearing a specific nucleotide sequence. Each input DNA strand was attached with a UMI by 2 PCR cycles. Further PCR amplification was performed after removing non-extended primers bearing UMI. NGS reads originating from the same input DNA strand carry the same UMI sequence, thus unique UMI family count represents the number of input DNA strands. b. Quantitation of ERBB2 and EIF2C1 molecule count using 2-plex QASeq and ddPCR. Five replicated experiments were performed for both methods with $10 \mathrm{ng}$ gDNA input. QASeq showed higher conversion yield ( $86 \%$ on average) than ddPCR ( $53 \%$ on average). The CV of molecule count was lower for QASeq $(5.0 \%$ for ERBB2, $2.5 \%$ for EIF2C1) than for ddPCR (12.8\% for ERBB2, 13.3\% for EIF2C1). c. Absolute quantitation of different DNA input. Observed ERBB2 molecule counts by QASeq were close to expected molecule counts calculated from DNA input amount because of high conversion yield. Lower conversion yield at $1 \mathrm{ng}$ input DNA was possibly a result of material loss at low concentration. d. Technical variation of ERBB2 ploidy using 2plex QASeq. The mean of 5 replicates was 1.98 , which is close to normal ploidy of 2. e. UMI family size distribution and data processing. UMI family size follows log-normal distribution after removing small families. Family size cutoff was calculated as $5 \%$ of the mean of top 3 largest family size here. 

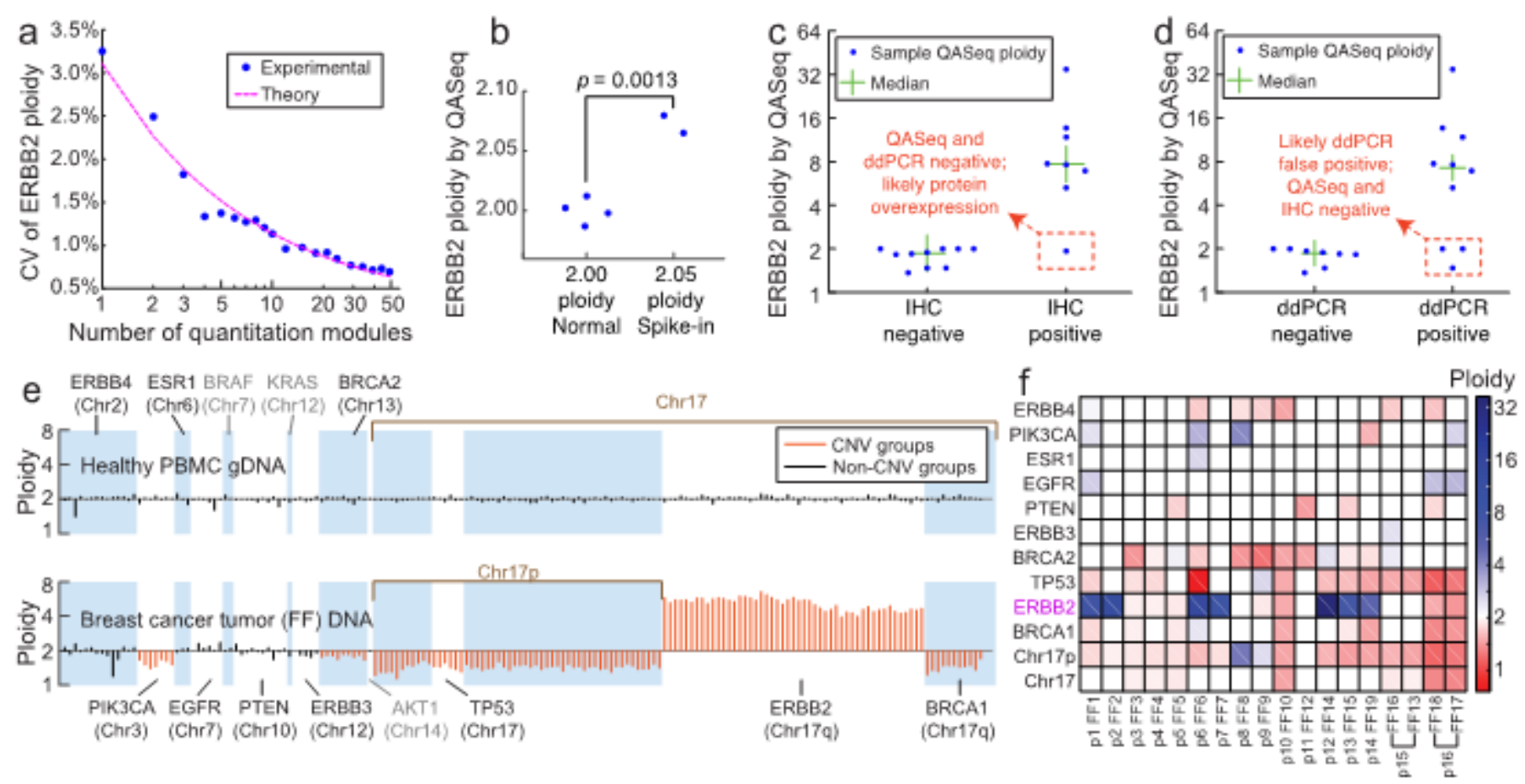

Figure 2

Combination of multiple absolute quantitation modules for CNV detection. a. Stochastic error in copy number quantitation was reduced by increasing the number of quantitation modules in the gene. ERBB2 ploidy was calculated as 2 times the ratio between the mean of UMI family counts from modules in ERBB2 and in the reference. Because there are multiple possibilities of module down-selection, here each datapoint represents the average of 30 randomized selections. 123 modules served as the reference for ploidy calculation. The CV of 1 module was lower than that in Fig. 1c, because only 1 module was used for both the reference and ERBB2. b. Discriminating 2.05 and 2.00 ploidy ERBB2 samples using QASeq. The P-value calculated using the two-sided t-test was 0.0013. c. Concordance of QASeq ERBB2 ploidy with IHC. For the sample with discordance between QASeq and IHC, ddPCR agreed with QASeq; this might be a result of ERBB2 over-experession at protein level due to changes in promoters or enhancers. $d$. Concordance of QASeq ERBB2 ploidy with ddPCR. For the 3 samples with discordance between QASeq and ddPCR, IHC agreed with QASeq. e. Ploidy of each QASeq quantitation module for a healthy PBMC donor (top) and for a breast cancer fresh/frozen (FF) tumor section (bottom). Genes with called CNVs are displayed in orange, genes within expected variation are shown in black. f. QASeq CNV calls for 18 fresh/frozen breast tumor sections. Two non-adjacent tumor sections were tested from patients $\mathrm{p} 15$ and p16. Between 5 and 8 ng DNA input was used based on availability. 

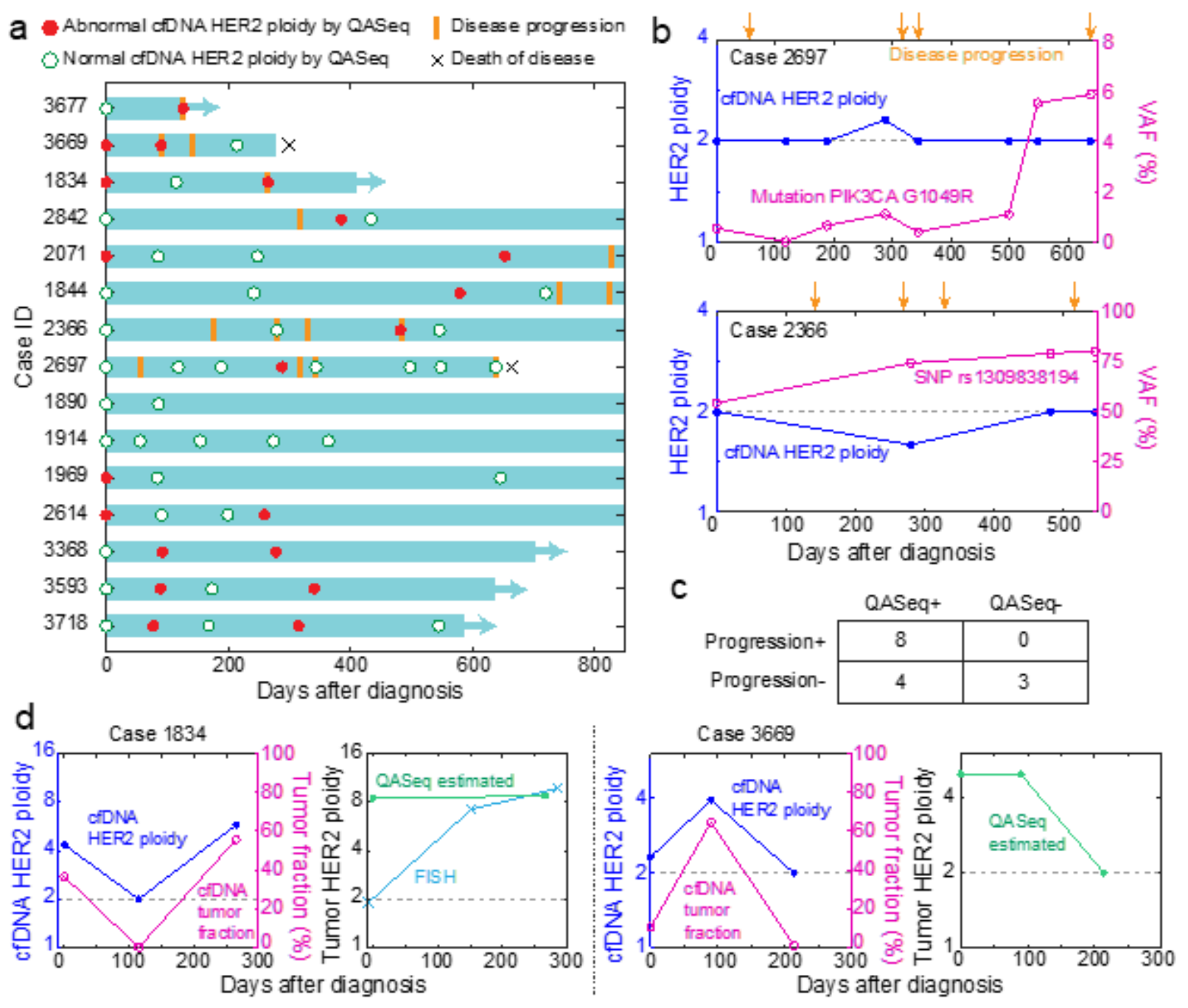

\section{Figure 3}

QASeq for longitudinal study of plasma samples from 15 ERBB2+ metastatic breast cancer patients. a. Swimmer plot of clinical course and molecular findings of patients. QASeq identified cfDNA ERBB2 amplification or increase of ERBB2 ploidy relative to the previous point in 6 out of the 8 patients with progression within 6 months of plasma sampling. The other 2 patients (sample ID 2697 and 2366) were each reported with 4 progressions. Although ERBB2 amplification or increase of ERBB2 ploidy was also observed in these two patients, abnormal ERBB2 events are only associated with part of the progression. b. ERBB2 ploidy and mutation allele frequency change in plasma sample of patients 2697 and 2366 . Significant allele frequency changes in PIK3CA G1049R mutation in patient 2697 and in SNP rs1309838194 in patient 2366 were correlated with progression. c. Categorization of patients based on QASeq abnormal molecular findings and disease progression. Chi-square test suggested that the QASeq result and progression is not statistically independent $(p=0.038)$. $d$. Plasma ERBB2 ploidy normalization with tumor fraction to infer tumor ERBB2 ploidy. Tumor FISH results at three time points were collected in patient 1834. QASeq detected ERBB2 amplification in plasma 5 months earlier than FISH from tumor tissue. The inferred tumor ERBB2 ploidy by QASeq was consistent with the available tumor ploidy from FISH. The inferred tumor ERBB2 ploidy was generally stable in both of the two patients 

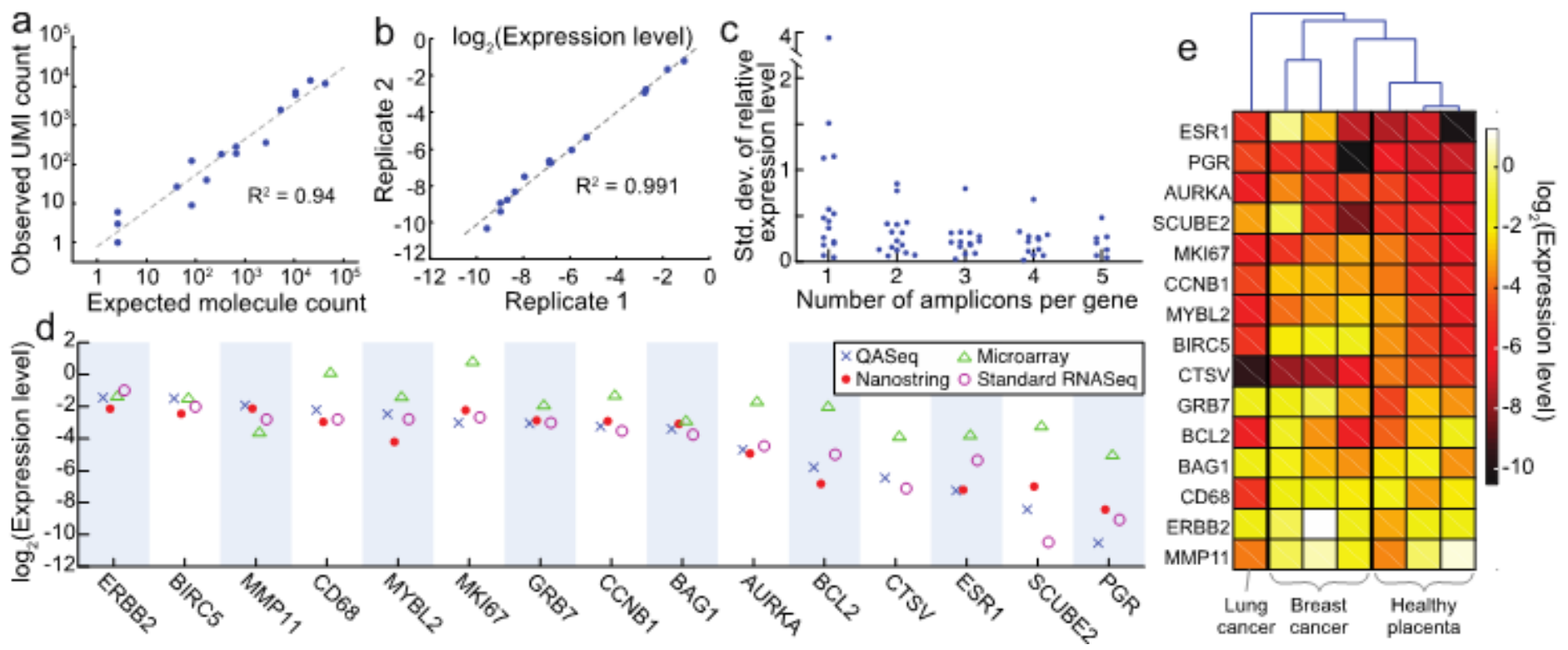

Figure 4

RNA QASeq for gene expression level quantitation. a. Quantitation accuracy validation with ERCC spike-in reference sample. b. RNA QASeq quantitation reproducibility in technical replicates. $10 \mathrm{ng}$ total liver RNA was used as input. c. Multiple amplicons per gene reduced quantitation variability in technical triplicate analysis of total liver RNA sample. Number of amplicons $=\mathrm{N}$ means only using the $\mathrm{N}$ amplicons at 5 ' end of mRNA. d. RNA relative expression level quantitation side-by-side comparison using four different methods in one breast cancer FFPE RNA sample. e. Relative expression level measured by RNA QASeq in four clinical FFPE and 3 normal placenta FFPE samples. Hierarchical clustering indicated the expression patterns were the most similar between normal placenta samples.

\section{Supplementary Files}

This is a list of supplementary files associated with this preprint. Click to download.

- QASeqSIfigdatanew.xlsx

- QASeqSISection6.xIsx

- QASeqSIsectionsv2.docx 